\title{
A Collocation Method for Quadratic Control Problems Governed by Ordinary Elliptic Differential Equations
}

\section{Walter Alt, Nils Bräutigam, Domnik Karolewski}

Institute for Applied Mathamatics, Friedrich-Schiller-University, Jena, Germany, alt@minet.uni-jena.de Keywords: Linear quadratic optimal control problems, elliptic equations, collocation method, error estimates

\section{INTRODUCTION}

We consider the one-dimensional elliptic optimal control problem

(CP) $\min \frac{1}{2} \int_{0}^{T}\left|z(t)-z_{d}(t)\right|^{2}+\nu|u(t)|^{2} d t$

s.t.

$-\ddot{z}(t)+A z(t)=B u(t)+e(t)$ for a.a. $t \in[0, T]$, $z(0)=z(T)=0$,

$a \leq u(t) \leq b$ for a.a. $t \in[0, T]$,

where $u \in L_{2}\left(0, T ; \mathbb{R}^{m}\right), z, z_{d} \in W_{2}^{2}\left(0, T ; \mathbb{R}^{n}\right)$, $\dot{e} \in B V\left(0, T ; \mathbb{R}^{n}\right), A \in \mathbb{R}^{n \times n}$ is symmetric and positive semidefinite, $B \in \mathbb{R}^{n \times m}$ and $a, b \in \mathbb{R}^{m}$, $a<b$.

\section{DISCRETIZATION OF THE STATE EQUATION}

For the discretization of the state equation

$-\ddot{z}(t)+A z(t)=y(t)$ for a.a. $t \in[0, T]$,

$z(0)=z(T)=0$,

we use a uniform grid

$$
G=\left\{t_{i}=i h \mid i=0, \ldots, N\right\}
$$

with mesh size $h=T / N, N \geq 2$. By $S_{h}=$ $S_{h}(y)$ we denote the quadratic spline with knots $t_{i}, i=0, \ldots, N$, defined by the collocation and boundary conditions

$$
\begin{aligned}
& -\ddot{S}_{h}\left(t_{i}\right)+A S_{h}\left(t_{i}\right)=y\left(t_{i}\right), i=0, \ldots, N, \\
& S_{h}(0)=S_{h}(T)=0 .
\end{aligned}
$$

If $z$ ist the solution of the state equation (1) and $\dot{y} \in B V\left(0, T ; \mathbb{R}^{n}\right)$, then

$$
\left\|z-S_{h}\right\|_{\infty} \leq c h^{2}
$$

with a constant $c$ independent of $h$ (see Sendov (5), Theorem 7.3).

\section{DISCRETIZATION OF THE CONTROL PROBLEM}

We define $U_{\text {ad }}=\left\{u \in L_{2}\left(0, T ; \mathbb{R}^{m}\right) \mid a \leq\right.$ $u(t) \leq b$ for a.a. $t \in[0, T]\}$. For a function $f$ continuous on $[0, T]$ we define

$$
\|f\|_{h}=\sqrt{h \sum_{i=0}^{N}\left|f\left(t_{i}\right)\right|^{2}} .
$$

Let $V_{h}\left(0, T ; \mathbb{R}^{m}\right)$ be the space of continuous, piecewise linear functions on the grid (2). Using the operator $\mathcal{S}_{h}$ we discretize problem (CP) in the following way:

$(\mathrm{CP})_{h} \min \frac{1}{2}\left\|\mathcal{S}_{h}\left(B u_{h}+e\right)-z_{d}\right\|_{h}^{2}+\nu\left\|u_{h}\right\|_{h}^{2}$

s.t. $u_{h} \in U_{h}^{a d}=U_{\mathrm{ad}} \cap V_{h}\left(0, T ; \mathbb{R}^{m}\right)$.

Problem $(\mathrm{CP})_{h}$ has a unique solution $\bar{u}_{h}$.

\section{ERROR ESTIMATES}

First we derive a result on discrete quadratic convergence for the solutions $\bar{u}_{h} \in V_{h}\left(0, T ; \mathbb{R}^{m}\right)$ of the problems $(\mathrm{CP})_{h}$.

Theorem 1. Let $\bar{u}$ be the solution of (CP2) with $\dot{\bar{u}} \in B V\left(0, T ; \mathbb{R}^{m}\right)$ and $\bar{u}_{h} \in V_{h}\left(0, T ; \mathbb{R}^{m}\right)$ the solution of the discrete problem $(\mathrm{CP})_{h}$. Then

$$
\left\|\bar{u}-\bar{u}_{h}\right\|_{h} \leq c h^{2}
$$

holds true with a constant $c$ independent of $h$.

The continuous error $\left\|\bar{u}-\bar{u}_{h}\right\|_{\infty}$ is only of order $3 / 2$. Therefore, we adopt the idea of Meyer/Rösch (4) (see also (1), (2)) to construct a new feasible control by

$$
\tilde{u}_{h}=\Pi_{[a, b]}\left(-\frac{1}{\nu} B^{\top} p_{h}\left(\bar{u}_{h}\right)\right),
$$

for which we can prove continuous convergence of order 2 . 
Theorem 2. Let $\bar{u}$ be the solution of problem (CP2) with $\dot{\bar{u}} \in B V\left(0, T ; \mathbb{R}^{m}\right)$ and $\bar{u}_{h} \in$ $V_{h}\left(0, T ; \mathbb{R}^{m}\right)$ the solution of the discrete problem $(\mathrm{CP})_{h}$. Then for the control $\tilde{u}_{h}$ defined by (4) we have the continuous error estimate

$$
\left\|\bar{u}-\tilde{u}_{h}\right\|_{\infty} \leq c h^{2}
$$

with a constant $c$ independent of $h$.

\section{REFERENCES}

[1] Alt W., Bräutigam N., Rösch A. (2007) Error Estimates for Finite Element Approximations of Elliptic Control Problems. To appear in Discussiones Mathematicae: Differential Inclusions, Control and Optimization.

[2] Alt W., Bräutigam N., Rösch A. (2007) FiniteDifference Discretizations of Quadratic Control Problems Governed by Ordinary Elliptic Differential Equations. To appear in Comp. Opt. Appl.

[3] Dontchev A. L., Hager W. W., Veliov V. V. (2000) Second-order Runge-Kutta Approximations in Control Constrained Optimal Control. SIAM J. Numer. Anal., 38, 202-226.

[4] Meyer C., Rösch A. (2004): Superconvergence Properties of Optimal Control Problems. SIAM J. Control and Optimization, 43, 970-985.

[5] Sendov B., Popov V. A. (1988) The Averaged Moduli of Smoothness. Wiley-Interscience. 